\title{
Invasive Pulmonary Aspergillosis Mimicking Cyst Hydatics
}

\author{
İsmail Agababaoglu*,[MD] \\ Hasan Ersöz ${ }^{1},[\mathrm{MD}]$ \\ Filiz Banu Çetinkaya Ethemoglu ${ }^{2},[\mathrm{MD}]$ \\ Aydın Şanlı ${ }^{3},[\mathrm{MD}]$ \\ ${ }^{1}$ Department of Thoracic Surgery, Katip Çelebi \\ University, İzmir/TURKEY \\ ${ }^{2}$ Department of Anesthesiology and Reanimation, \\ Yıldırım Beyazıd University Yenimahalle Education and \\ Research Hospital, Ankara/TURKEY \\ ${ }^{3}$ Department of Thoracic Surgery, Dokuz Eylül \\ University, İzmir/TURKEY,
}

\section{reo ABSTRACT Cen}

Invasive pulmonary aspergillosis is a less frequent form and rarely has been reported in normal immune system cases. They do not constitute radiologically cystic structures. A 53-year-old male who was engaged in stock farming and agriculture has no additional disease or habit in the patient history that would compromise the immune system. In thorax computed tomography, a $11 \times 8 \times 10 \mathrm{~cm}$ diaphragmatic invasive cystic lesion was seen in the middle and lower lobes of the right lung, It was reported that there may be hydatid cyst. The patient underwent right lower bilobectomy and diaphragm resection, the diaphragm was reconstructed with a dual mesh. Histopathologic diagnosis was reported as invasive pulmonary aspergillosis. There was no complication or recurrence in the case in the 24-month follow-up period. Thus, a cure was provided for our patient with invasive pulmonary aspergillosis, which was coincidentally diagnosed by pathology in the postoperative period.

Keywords: Invasive pulmonary aspergillosis, hydatid cyst, surgery

\author{
* Correspondence author: İsmail Ağababaoğlu, \\ Department of Thoracic Surgery, Yıldırım Beyazıd \\ University Yenimahalle Education and Research \\ Hospital, Ankara/TURKEY \\ Phone number: +90 3125872141 \\ Fax number: +90 3125872437 \\ email: ismailagababaoglu@gmail.com
}

Received: 10 Agust 2019, Accepted: 11 September 2019,

Published online: 30 September 2019

\section{INTRODUCTION}

Aspergillus is a fungus commonly found in nature. The inhalation of spores can be easily eliminated when the immune system is normal. However, inhalation of spores during immune-compromised cases may result in different clinical manifestations; aspergilloma, chronic necrotizing aspergillosis, invasive pulmonary aspergillosis (IPA), etc. IPA is less frequent form and rarely has been reported in normal immune system cases. They don't constitute radiologically cystic structures. In this respect, we present this unexampled rare case, in which we administered surgical treatment taking into consideration the hydatid cyst.

\section{CASE REPORT}

A 53-year-old male applied to the hospital with chest pain and cough. The patient's complaints are acute onset and the patient has no previous follow-up due to any disease. The patient had no hemoptysis at the time of admission to the emergency department. No disease in patient history would compromise the immune system. The chest $x$-ray showed a cystic lesion in the right lower zone. In thorax computed tomography, a $11 \times 8 \times 10 \mathrm{~cm}$ diaphragmatic invasive cystic lesion was seen in the middle and lower lobes that reported hydatid cyst (Figure 1). The preoperative anti-echinococcus IgG and IgM indrect hemagglutination test and other serological tests were not performed because of low diagnostic 
value [1]. The patient underwent posterolateral thoracotomy under elective conditions. Lung parenchyma was released from the chest wall. It was observed that the lesion was invasive to the diaphragm. Anatomic resection was decided because the cyst is observed in more than $50 \%$ of the middle and lower lobes [1]. Pulmonary function test was performed before the operation and forced-expiratory-volume in one-second value was $2200 \mathrm{ml} / \mathrm{sec}(\% 85)$. This result indicated that the patient had enough respiratory reserve for the planned resection. The patient underwent a right lower bilobectomy. During this time, the diaphragm was resected without opening the cyst and it was taken out as unblocked together with the lobectomy material. Then, the diaphragm was reconstructed with a dual mesh. The preoperative frozen section was not worked because it would not change our surgical strategy. Postoperatively, because the right upper lobe didn't fill sufficiently the thorax, a pouch formed in the lower half of the right hemithorax. The patient's thoracic drainage was terminated on the seventh postoperative day. Complications; Emphysema, air leak, hemorrhage, etc. in the early postoperative period were not consisted of. Histopathologic diagnosis was reported as invasive pulmonary aspergillosis (Figure 2). After reaching the pathology result, chest and infectious diseases department have evaluated the patient for immunodeficiensy but no additional pathology was detected. Since invasive pulmonary aspergillosis was not considered in the early diagnosis, blood culture was not taken and transthoracic needle biopsy and bronchoscopy were not performed because the cyst was considered as a hydatid cyst. In blood and pleural fluid cultures studied postoperatively, reproduction was not detected. No reproduction was detected in bronchial lavage cultures obtained with fiberoptic bronchoscopy and galactomannan was negative. Surgical resection applied in the case was accepted as curative and additional treatment was not recommended. There was no complication or recurrence in the case in the 24-month follow-up period.

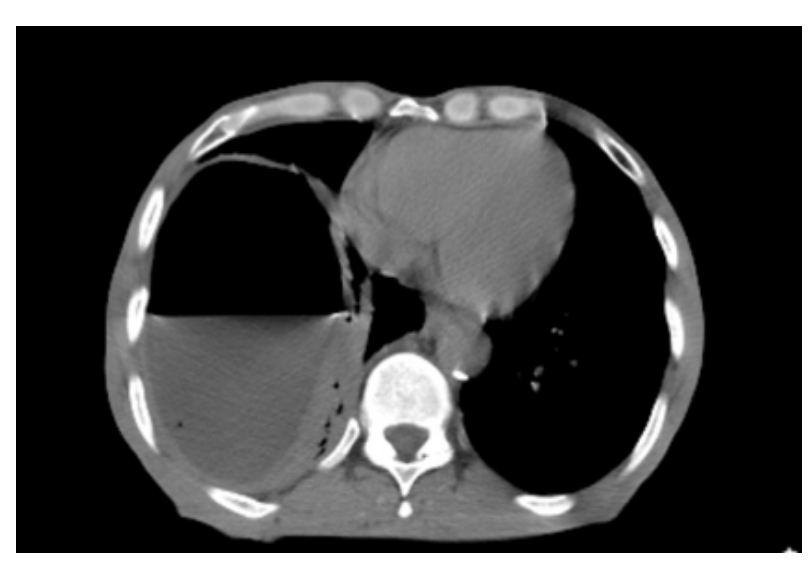

Figure 1.Thorax computed tomography view
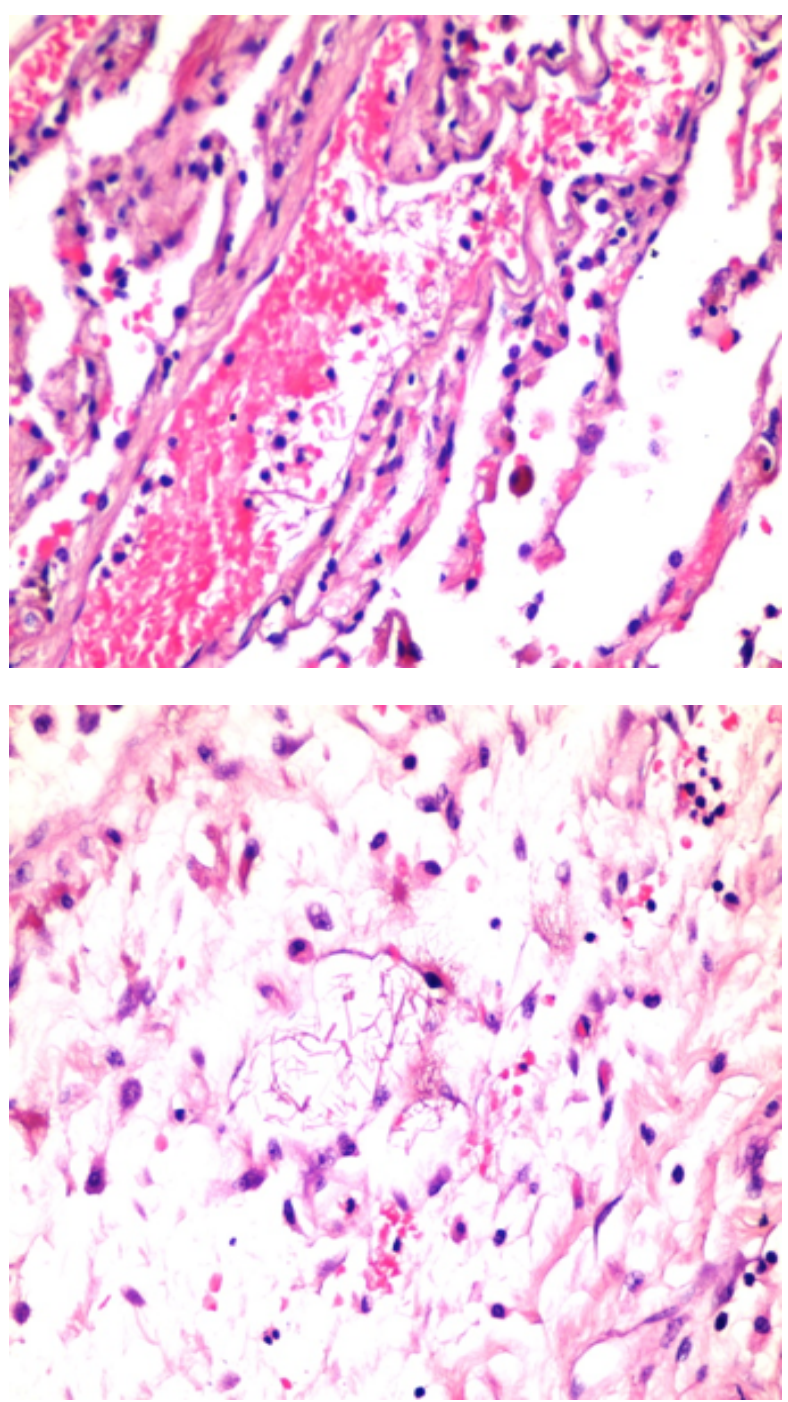

Figure 2. Pathological view 


\section{DISCUSSION}

Aspergillus fungus is a pathogen that can be seen around the world. Under normal conditions, when the immune system is normal, it does not cause any pathology colonizing especially in the respiratory tract. However, the prevalence of the use of immunocompromising drugs has increased due to the increased use of inhaled steroids $[2,3]$. Aspergilloma can lead to different clinical manifestations such as chronic necrotizing aspergillosis, invasive pulmonary aspergillosis, allergic bronchopulmonary aspergillosis. Invasive pulmonary aspergillosis among these clinical manifestations is characterized by colonization of the Aspergillus spores in lung parenchyma and their progression to necrosis [4]. Especially those inside the cavity are life-threatening because they cause sudden and massive hemoptysis [5]. In the diagnosis of invasive pulmonary aspergillosis, it is important to keep in mind that the condition may compromise the immune system and may be an Aspergillus infection [6]. Simple sputum culture, radiology may be helpful in the diagnosis, and it is reported that definite diagnosis was made by the detection of Aspergillus or hyphae in biopsy materials obtained with transthoracic, transbronchial needle aspirations or thoracotomies [7]. Our case here did not have anything to compromise the immune system. There was no long-term use of inhaled corticosteroids due to asthma or chronic obstructive pulmonary disease. This is the most important reason for not predicting invasive pulmonary aspergillosis in the early diagnosis.

Radiology may help diagnose invasive pulmonary aspergillosis. On chest $\mathrm{x}$-rays and thorax computerized tomography, single or multiple nodules and lesions are seen as an early finding. In advanced cases, there are patchy lesions in necrotic areas. Some infiltrations can interfere with pneumonia. In some of the cases, the cavities involving a fungus ball may form lesions during the healing process. It had not been previously reported that invasive pulmonary aspergillosis, as in our case, is manifested by the cystic structure. Because of this, firstly the cyst was considered as hydatid since our case was engaged in livestock and agriculture and living in the endemic region. An operation was planned because curative treatment in lung cyst hydatic cases was surgery [1]. Since the transthoracic needle biopsy and bronchoscopy may cause the hydatid cysts to be ruptured, these procedures were avoided. Anatomic resection was preferred to parenchymal preserving surgery since cyst is observed in more than $50 \%$ of the hydatid lobe, diaphragm reconstruction was performed with right lower lobectomy, diaphragmatic resection, and dual mesh. Thus, a cure was provided for our patient with invasive pulmonary aspergillosis, which was coincidentally diagnosed by pathology in the postoperative period.

There are medical drugs that can be used in cases of invasive pulmonary aspergillosis. However, it has been reported that medical treatments are inadequate to completely eradicate the infection [8]. In this respect, some publications support the surgical approach in the treatment of invasive pulmonary aspergillosis cases. The surgical approach in these studies is suggested especially in cases of possible complications such as residual tissues after medical treatment, cases accompanied by malignancy, and hemorrhage [9,11]. Apart from treatment, reports are suggesting an invasive surgical approach to invasive pulmonary aspergillosis at the diagnosis stage [12]. However, there are also studies in the literature which show that the morbidity and mortality of the surgery are very high and object to the surgeries [13]. In our case, even if invasive pulmonary aspergillosis had been considered instead of hydatid cyst in the early diagnosis, surgery would be indicated because the lung is a damaged lung that cannot function again and a hemorrhagic complication risk is high.

As a result, although invasive pulmonary aspergillosis is mostly seen in immunocompromised cases and does not give a bulky cystic image in radiological evaluations, it should be remembered in the cases with the normal immune system, radiological imaging and early diagnosis of cases with cystic lesions as in our case. Moreover, regarding such cases, there is no definite information about the diagnosis and the necessary treatment approach in the literature. In cases with invasive pulmonary aspergillosis, as manifested in our case, although cases that can tolerate surgery are asymptomatic, surgery should be preferred in treatments to prevent the possible life-threatening complications and any invasive diagnostic procedures that may complicate the case should be avoided.

\section{CONFUCTS of INTEREST}

There is no conflict of interest. 
[1] Özyurtkuran MO, Balcı AE. Surgical treatment of intrathoracic hydatid disease: A 5 five year experience in an endemic region. SurgToday 2010; 40: 31-37.

[2] Lionakis MS, Kontoyiannis DP. Glucocorticoids and invasive fungal infections. Lancet 2003; 362: 1828-38.

[3] Fukuda Y, Homma T, Suzuki S, et al. High burden of Aspergillus fumigatus infection among chronic respiratory diseases. Chron Respir Dis 2018; 15(3): 279-85. doi: $10.1177 / 1479972318761654$.

[4] Hope WW, Walsh TJ, Denning DW. The invasive and saprophytic syndromes due to Aspergillus spp. Med Mycol 2005; 43: Suppl. 1, S207-S238.

[5] Harmouchi H, Lakranbi M, Issoufou I, et al. Pulmonary aspergilloma: surgical outcome of 79 patients in a Moroccan center. Asian Cardiovasc Thorac Ann 2019; 6- 5: 218492319855492. doi: 10.1177/0218492319855492.

[6] Walsh TJ, Roilides E, Cortez K, Kottilil, et al. Control, immuno regulation, and expression of innate pulmonary host defenses agains tAspergillus fumigatus. Med Mycol 2005; 43(suppl 1): S165-72. 1, S165-S172.

[7] Scott SM, Takaro T. Thoracic Infections Caused by Actinomycetes, Fungi, opportunistic organisms, and Echinococcus. In: Bauer AA, Geha AS, Hammond GL, et al (eds). Glenn's Thoracic and Cardiovascular Surgery. 5th ed.
SA; Prentice Hail Int. Inc, 1991: 273-5. Hail International Inc. 1991:273-5. 273-275

[8] Maertens J, Raad I, Petrikkos G, et al. Efficacy and safety of caspofungin for treatment of invasive aspergillosis in patients refractory or intolerant of conventional antifungal therapy. ClinInfectDis 2004; 39: 1563-1571.

[9] Salerno CT, Ouyang DW, Pederson TS, et al. Surgical therapy for pulmonary aspergillosis in immuno compromised patients. Ann Thorac Surg 1998; 65: 1415-1419.

[10] Reichenberger F, Habicht J, Kaim A, et al. Lung resection for invasive pulmonary aspergillosis in neutropenic patients with hematologic diseases. Am J RespirCritCareMed. 1998; 158: 885-90

[11] Pidhorecky I, Urschel J, Anderson T. Resection of invasive pulmonary aspergillosis in immuno compromised patients. AnnSurgOncol. 2000; 7: 312-17

[12] Caillot D, Casasnovas O, Bernard A, et al. Improved management of invasive pulmonary aspergillosis in neutropenic patients using early thoracic computed tomographic scan and surgery. J Clin Oncol 1997; 15: 139-47

[13] Shirakusa $T$, Ueda $H$, Suito $T$, et al. Surgical treatment of pulmonary aspergilloma and aspergillus empyema. Ann Thorac Surg 1989, 48: 779-82. 